\title{
On the existence of a gap in the energy spectrum of quantum systems
}

\author{
W J Caspers $†$ and W Magnus $\ddagger \S$ \\ † Twente University of Technology, Enschede, The Netherlands \\ $\ddagger$ Instituut voor Theoretische Fysika, University of Leuven, B-3030 Leuven, Belgium
}

Received 27 June 1983

\begin{abstract}
In this paper a theorem on the existence of a gap in the energy spectrum of quantum systems, the exact ground state of which is known explicitly, is proved. The theorem is applied to a three-dimensional Heisenberg spin- $\frac{1}{2}$ ferromagnet, with anisotropic nearestneighbour interactions, and to an alternating Heisenberg antiferromagnet, with nearestand next-nearest-neighbour interactions.
\end{abstract}

\section{Definitions and notations}

Consider a quantum system described by a Hamiltonian $H$. The exact ground state(s) are supposed to be known explicitly. Let

$$
H=H_{1}+H_{2}
$$

where both $H_{1}$ and $H_{2}$ have at least one common ground state, which is consequently also a ground state of $H$. We use the following notation:

H Hilbert space of state vectors,

$n \operatorname{dim} \mathscr{H}$,

$\alpha \quad$ index which runs over 1,2 ,

$\mathscr{H}_{0 \alpha} \quad$ subspace spanned by all ground states of $H_{\alpha}$,

$E_{0 \alpha}$ ground-state energy of $H_{\alpha}$,

$\mathscr{H}_{0}$ subspace, spanned by all common ground states of $H, H_{1}$ and $H_{2}$,

$E_{0} \quad$ ground-state energy of $H$,

$\overline{\mathscr{H}}_{0 \alpha} \quad$ set of all ground states of $H_{\alpha}$ only,

$\mathscr{H}_{1 \alpha}$ orthogonal complement of $\mathscr{H}_{0 \alpha}$ in $\mathscr{H}$,

$|\psi\rangle \quad$ lowest-lying excited state of $H$.

The state $|\psi\rangle$ has the following properties:

$$
H|\psi\rangle=E_{1}|\psi\rangle \quad E_{1}>E_{0} \quad|\psi\rangle \perp \mathscr{H}_{0}
$$

and can thus be written:

$$
|\psi\rangle=a\left|\varphi_{01}\right\rangle+b\left|\varphi_{11}\right\rangle \quad\left|\varphi_{01}\right\rangle \in \overline{\mathscr{H}}_{01} \quad\left|\varphi_{11}\right\rangle \in \overline{\mathscr{H}}_{11}
$$

$\S$ Onderzoeker IIKW Belgium.

(C) 1983 The Institute of Physics 
where

$$
|a|^{2}+|b|^{2}=1
$$

if all state vectors are normalised to unity. Before the theorem is formulated, some trivial but important remarks should be made:

1.1. $\mathscr{H}_{0}=\mathscr{H}_{01} \cap \mathscr{H}_{02}$

Let $\left|\phi_{0}\right\rangle \in \mathscr{H}_{01} \cap \mathscr{H}_{02}$. Then $H_{\alpha}\left|\varphi_{0}\right\rangle=E_{0 \alpha}\left|\varphi_{0}\right\rangle, \alpha=1,2$ whereas

$$
H\left|\varphi_{0}\right\rangle=\left(E_{01}+E_{02}\right)\left|\varphi_{0}\right\rangle .
$$

Using the Rayleigh-Ritz variational principle, we get:

$$
E_{0} \leqslant E_{01}+E_{02} \text {. }
$$

On the other hand, $E_{01}+E_{02}$ is a lower bound for the lowest eigenvalue of $H_{1}+H_{2}$, whence

$$
E_{0} \geqslant E_{01}+E_{02} \text {. }
$$

Therefore we have:

$$
E_{0}=E_{01}+E_{02} \quad H\left|\varphi_{0}\right\rangle=E_{0}\left|\varphi_{0}\right\rangle,
$$

which proves $\mathscr{H}_{01} \cap \mathscr{H}_{02} \subset \mathscr{H}_{0}$.

On the other hand, let $\left|\varphi_{0}\right\rangle \notin \mathscr{H}_{01} \cap \mathscr{H}_{02}$, say $\mid \varphi_{0} \notin \mathscr{H}_{01}$. Then:

$$
\left\langle\varphi_{0}\left|H_{1}\right| \varphi_{0}\right\rangle>E_{01} \text {. }
$$

Because $\left\langle\varphi_{0}\left|H_{2}\right| \varphi_{0}\right\rangle$ is an upper bound for $E_{02}$, we also have:

$$
\left\langle\varphi_{0}\left|H_{2}\right| \varphi_{0}\right\rangle \geqslant E_{02} \text {. }
$$

From (2) and (3):

$$
\left\langle\varphi_{0}\left|\left(H_{1}+H_{2}\right)\right| \varphi_{0}\right\rangle>E_{01}+E_{02}=E_{0} .
$$

$\left|\varphi_{0}\right\rangle$ can thus never be a ground state of $H:\left|\varphi_{0}\right\rangle \notin \mathscr{H}_{0}$.

This proves $\mathscr{H}_{0} \subset \mathscr{H}_{01} \cap \mathscr{H}_{02}$ and consequently:

$$
\mathscr{H}_{0}=\mathscr{H}_{01} \cap \mathscr{H}_{02} \text {. }
$$

1.2 .

From the definitions of $\mathscr{H}_{0}, \overline{\mathscr{H}}_{0 \alpha}, \mathscr{H}_{1 \alpha}$, it follows straightaway that:

$\mathscr{H}=\mathscr{H}_{0} \oplus \overline{\mathscr{H}}_{0 \alpha} \oplus \mathscr{H}_{1 \alpha} \quad \overline{\mathscr{H}}_{0 \alpha} \perp \mathscr{H}_{0} \quad \mathscr{H}_{1 \alpha}+\overline{\mathscr{H}}_{0 \alpha} \quad \mathscr{H}_{1 \alpha} \perp \mathscr{H}_{0} \quad \alpha=1,2$.

It should be mentioned, however, that in general, $\overline{\mathscr{H}}_{01} \perp \overline{\mathscr{H}}_{02}$.

\section{Theorem on the existence of an energy gap in the spectrum of quantum systems}

If there exists a unitary operator $T$, satisfying

$$
[H, T]=0 \quad T H_{1} T^{+}=H_{2} \quad T H_{2} T^{-}=H_{1}
$$


and if there exists a number $x \in[0,1]$, such that

$$
\left|\left\langle\varphi_{01}|T| \varphi_{01}\right\rangle\right| \leqslant x
$$

then

$$
E_{1}-E_{0} \geqslant(1-x) \Delta
$$

where $\Delta$ is the common energy gap in the spectra of $H_{1}$ and $H_{2}=T H_{1} T^{+}$.

Proof: Because of $[H, T]=0,|\psi\rangle$ may be chosen to be a simultaneous eigenvector of $H$ and $T$ :

$$
H|\psi\rangle=E_{1}|\psi\rangle \quad T|\psi\rangle=\mathrm{e}^{\mathrm{i} q}|\psi\rangle \quad q \text { being real. }
$$

Following (1) and (4) we may also write:

$$
\begin{gathered}
\mathrm{e}^{\mathrm{i} q}|\psi\rangle=T|\psi\rangle=a T\left|\varphi_{01}\right\rangle+b T\left|\varphi_{11}\right\rangle \\
|\psi\rangle=\mathrm{e}^{-\mathrm{i} q} a T\left|\varphi_{01}\right\rangle+\mathrm{e}^{-\mathrm{i} q} b T\left|\varphi_{11}\right\rangle .
\end{gathered}
$$

Putting

$$
T_{00}=\left\langle\varphi_{01}|T| \varphi_{01}\right\rangle \quad T_{01}=\left\langle\varphi_{01}|T| \varphi_{11}\right\rangle
$$

we multiply both sides of (5) by $\left\langle\varphi_{01}\right|$ to obtain:

$$
\left(1-\mathrm{e}^{-\mathrm{i} q} T_{00}\right) a-\mathrm{e}^{-\mathrm{i} q} T_{01} b=0 .
$$

This equation is solved for $b / a$ :

$$
b / a=\left(\mathrm{e}^{\mathrm{i} q}-T_{00}\right) / T_{01} .
$$

Thus:

$$
\frac{|b|}{|a|}=\frac{\left|\mathrm{e}^{\mathrm{i} q}-T_{00}\right|}{\left|T_{01}\right|} \geqslant \frac{1-\left|T_{00}\right|}{\left|T_{01}\right|} .
$$

Using (6), we observe that $\left(\left|T_{00}\right|^{2}+\left|T_{01}\right|^{2}\right)^{1 / 2}$ is simply the norm of the projection of $\left|\varphi_{01}\right\rangle$ onto the manifold, spanned by the two orthonormal vectors $T\left|\varphi_{01}\right\rangle$ and $T\left|\varphi_{11}\right\rangle$, and therefore cannot exceed its upper bound 1 :

$$
\left|T_{00}\right|^{2}+\left|T_{01}\right|^{2} \leqslant 1
$$

or

$$
1 /\left|T_{01}\right| \geqslant\left(1-\left|T_{00}\right|^{2}\right)^{-1 / 2}
$$

From (7) and (8), some useful inequalities are derived:

$$
\begin{aligned}
& \frac{|b|}{|a|} \geqslant \frac{1-\left|T_{00}\right|}{\left(1-\left|T_{00}\right|^{2}\right)^{1 / 2}}=\left(\frac{1-\left|T_{00}\right|}{1+\left|T_{00}\right|}\right)^{1 / 2} \\
& |b|^{2} \geqslant \frac{1-\left|T_{00}\right|}{1+\left|T_{00}\right|}|a|^{2} .
\end{aligned}
$$

Remembering $|a|^{2}+|b|^{2}=1$ and $\left|T_{00}\right| \leqslant x$, we find:

$$
|b|^{2} \geqslant \frac{1}{2}\left(1-\left|T_{00}\right|\right) \geqslant \frac{1}{2}(1-x) .
$$


Now,

$$
E_{1}=\langle\psi|H| \psi\rangle=2\left\langle\psi\left|H_{1}\right| \psi\right\rangle=2\left[|a|^{2} E_{01}+|b|^{2}\left\langle\varphi_{11}\left|H_{1}\right| \varphi_{11}\right\rangle\right] .
$$

Clearly, in this case, $E_{01}=E_{02}=\frac{1}{2} E_{0},\left\langle\varphi_{11}\left|H_{1}\right| \varphi_{11}\right\rangle \geqslant \frac{1}{2} E_{0}+\Delta$, from which we conclude:

$$
\begin{aligned}
& E_{1} \geqslant 2\left[|a|^{2} \frac{1}{2} E_{0}+|b|^{2}\left(\frac{1}{2} E_{0}+\Delta\right)\right] \\
& E_{1} \geqslant E_{0}+2|b|^{2} \Delta \\
& E_{1} \geqslant E_{0}+(1-x) \Delta .
\end{aligned}
$$

The last inequality is obtained by using (9).

Corollary: The simple condition $x<1$ does not give any information about the behaviour of $\left|\left\langle\varphi_{01}|T| \varphi_{01}\right\rangle\right|$ as a function of $n$. Now, because both $E_{0}$ and $E_{1}$ are extensive quantities, the theorem becomes only useful if $1-x$ always remains a finite positive number, no matter how large $n$ becomes. It has to be checked in every practical case whether this condition is fulfilled or not.

\section{Generalisations of the theorem}

In two cases a straightforward generalisation of the theorem can be given. The assumption that $H$ and its subdividing parts have at least one common ground state, remains valid.

\section{1.}

Let $H$ be subdivided into three equivalent parts, according to

$$
H=H_{1}+H_{2}+H_{3},
$$

where $T H_{1} T^{+}=H_{2}, T H_{2} T^{+}=H_{3}, T H_{3} T^{+}=H_{1}$, and $T$ is a unitary operator which generates a cyclic permutation of $H_{1}, H_{2}$ and $H_{3}$. The specific choice for $q(q=0$, $\pm 2 \pi / 3$ ) clearly does not change the course of the proof, so that (9) still holds.

Writing this time:

$E_{1}=\left\langle\psi\left|\left(H_{1}+H_{2}+H_{3}\right)\right| \psi\right\rangle=3\left\langle\psi\left|H_{1}\right| \psi\right\rangle=3\left[|a|^{2 \frac{1}{3}} E_{0}+|b|^{2}\left(\frac{1}{3} E_{0}+\Delta\right)\right]$

where $\Delta$ is again the common gap in the spectra of $H_{1}, H_{2}$ and $H_{3}$, we get:

$$
E_{1}-E_{0} \geqslant 3|b|^{2} \Delta \quad E_{1}-E_{0} \geqslant \frac{3}{2}(1-x) \Delta .
$$

3.2.

Let $H=H_{1}+H_{2}$, where $H_{2}$ is no longer a unitary transformation of $H_{1}$. Denoting by $\Delta_{\alpha}$ the gap in the energy spectrum of $H_{\alpha}(\alpha=1,2)$, and putting $\Delta=\min \left(\Delta_{1}, \Delta_{2}\right)$, we start again from equation (1):

$$
|\psi\rangle=a\left|\varphi_{01}\right\rangle+b\left|\varphi_{11}\right\rangle
$$

Because of $|\psi\rangle \perp \mathscr{H}_{0}$, we also may write an alternative expansion of $|\psi\rangle$, according to: $|\psi\rangle=c\left|\varphi_{02}\right\rangle+d\left|\varphi_{12}\right\rangle \quad\left|\varphi_{02}\right\rangle \in \overline{\mathscr{H}}_{02} \quad\left|\varphi_{12}\right\rangle \in \mathscr{H}_{12} \quad|c|^{2}+|d|^{2}=1$. 
Again, all state vectors are normalised to unity. Putting

$$
T_{00}=\left\langle\varphi_{01} \mid \varphi_{02}\right\rangle \quad T_{01}=\left\langle\varphi_{01} \mid \varphi_{12}\right\rangle \quad T_{10}=\left\langle\varphi_{11} \mid \varphi_{02}\right\rangle
$$

we multiply (1) by $\left\langle\varphi_{02}\right|$ and (12) by $\left\langle\varphi_{01}\right|$ to obtain:

$$
c=T_{00}^{*} a+T_{10}^{*} b \quad a=T_{00} c+T_{01} d .
$$

From (13) the basic inequalities are derived:

$$
\begin{aligned}
& |c| \leqslant\left|T_{00}\right||a|+\left|T_{10}\right||b| \\
& |a| \leqslant\left|T_{00}\right||c|+\left|T_{01}\right||d| .
\end{aligned}
$$

Therefore:

$\left[1-\left|T_{00}\right|^{2}\right]\left[|a|^{2}+|c|^{2}\right] \leqslant\left|T_{10}\right|^{2}|b|^{2}+\left|T_{01}\right|^{2}|d|^{2}+2\left|T_{00}\right|\left[\left|T_{10}\right||a||b|+\left|T_{01}\right||c||d|\right]$.

Projecting $\left|\varphi_{01}\right\rangle$ onto the manifold spanned by $\left|\varphi_{02}\right\rangle$ and $\left|\varphi_{12}\right\rangle$, and, conversely, projecting $\left|\varphi_{02}\right\rangle$ onto the manifold, spanned by $\left|\varphi_{01}\right\rangle$ and $\left|\varphi_{11}\right\rangle$, we get:

$$
\left|T_{00}\right|^{2}+\left|T_{01}\right|^{2} \leqslant 1 \quad\left|T_{00}\right|^{2}+\left|T_{10}\right|^{2} \leqslant 1 .
$$

Now, $|a|^{2}+|b|^{2}=|c|^{2}+|d|^{2}=1$ implies:

$$
|a||b| \leqslant \frac{1}{2} \quad|c||d| \leqslant \frac{1}{2} .
$$

After a straightforward calculation that makes use of (14), (15) and (16), we arrive at:

$$
\left.\left|b^{2}+\right| d\right|^{2} \geqslant 1-x /\left(1-x^{2}\right)^{1 / 2},
$$

provided that $\left|T_{00}\right| \leqslant x$.

$E_{1}$ is estimated as follows:

$$
\begin{aligned}
& E_{1}=\left\langle\psi\left|H_{1}\right| \psi\right\rangle+\left\langle\psi\left|H_{2}\right| \psi\right\rangle=|a|^{2} E_{01}+|c|^{2} E_{02}+|b|^{2}\left\langle\varphi_{11}\left|H_{1}\right| \varphi_{11}\right\rangle+|d|^{2}\left\langle\varphi_{12}\left|H_{2}\right| \varphi_{12}\right\rangle \\
& E_{1} \geqslant|a|^{2} E_{01}+|b|^{2}\left(E_{01}+\Delta_{1}\right)+|c|^{2} E_{02}+|d|^{2}\left(E_{02}+\Delta_{2}\right) \\
& E_{1}-E_{0} \geqslant|b|^{2} \Delta_{1}+|d|^{2} \Delta_{2} \geqslant\left[|b|^{2}+|d|^{2}\right] \Delta .
\end{aligned}
$$

So it follows in a straightforward way that:

$$
E_{1}-E_{0} \geqslant\left[1-x /\left(1-x^{2}\right)^{1 / 2}\right] \Delta .
$$

This version of the theorem, however, is only of practical use if $1-x /\left(1-x^{2}\right)^{1 / 2}$ is strictly positive, as can be seen from (18). This requires $x$ to be confined to the interval $[0, \sqrt{2} / 2[$.

\section{Applications}

In all examples we assume periodic boundary conditions to be valid.

\subsection{The anisotropic spin- $\frac{1}{2}$ Heisenberg ferromagnet in three dimensions}

The system is represented by the following Hamiltonian:

$$
H=-2 J \sum_{\left\langle\boldsymbol{R}, R^{\prime}\right\rangle}\left(S_{R}^{x} S_{R^{\prime}}^{x}+S_{R}^{y} S_{R^{\prime}}^{y}+\gamma S_{R}^{z} S_{R^{\prime}}^{z}\right) \quad J>0, \gamma>1 .
$$


In $\Sigma_{\left\langle R, R^{\prime}\right\rangle} \boldsymbol{R}, \boldsymbol{R}^{\prime}$ run over all $N$ sites of a simple cubic lattice, with the restriction that they always refer to a nearest-neighbour pair. If $a$ is the elementary lattice distance and if $e_{x}$, $\boldsymbol{e}_{y}, \boldsymbol{e}_{z}$ are chosen in such a way that $a \boldsymbol{e}_{x}, a \boldsymbol{e}_{y}, a \boldsymbol{e}_{z}$ correspond to the three elementary lattice translations, then $H$ can be written as

$$
\begin{aligned}
& H=\sum_{\delta=a e_{x}, a e_{1}, a e_{2}} H_{\delta}
\end{aligned}
$$

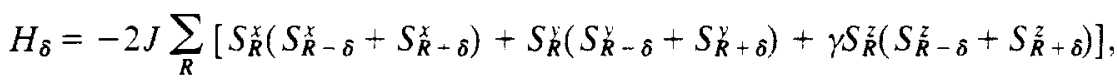

or

$$
H_{\delta}=-4 J \sum_{R}\left[S_{R}^{x} S_{R+\delta}^{x}+S_{R}^{\chi} S_{R-\delta}^{y}+\gamma S_{R}^{z} S_{R}^{z}-\delta\right] .
$$

Let $T(\delta)$ represents a unitary operator which generates a translation over $\delta$, and let $\boldsymbol{\rho}(\boldsymbol{\delta})$ run over all odd sites in the direction of $\boldsymbol{\delta}$, i.e.

$$
\begin{aligned}
& \boldsymbol{\rho}(\boldsymbol{\delta})=a\left[(2 n+1) \boldsymbol{e}_{x}+m \boldsymbol{e}_{y}+l \boldsymbol{e}_{z}\right] \text { for } \\
& \boldsymbol{\delta}=a \boldsymbol{e}_{x}, n, m, \text { and } l \text { being intergers, and so on. }
\end{aligned}
$$

Then

$H_{\delta}=H_{\delta}+H_{2 \delta}$

$H_{1 \delta}=4 J \sum_{\rho(\delta)}\left[S_{\rho(\delta)}^{x} S_{\rho(\delta)-\delta}^{x}+S_{\rho(\delta)}^{y} S_{\rho(\delta)+\delta}^{y}+\gamma S_{\rho(\delta)}^{z} S_{\rho(\delta)+\rho}^{z}\right]=\sum_{\rho(\delta)} H_{1 \rho(\delta)}$

$H_{2 \delta}=T(\delta) H_{1 \delta} T^{+}(\delta)$.

For completeness, we first check the two aligned states $\left|F_{+}\right\rangle$and $\left|F_{-}\right\rangle$(which have respectively all spins up and down with respect to $\boldsymbol{e}_{z}$ ) to be the ground states of $H$. For that purpose, we use the subdivision

$$
H=\sum_{\delta}\left(H_{1 \delta}+H_{2 \delta}\right),
$$

where both $H_{16}$ and $H_{2 \delta}$ describe $N / 2$ non-interacting spin pairs. Therefore, it is sufficient to show that the ferromagnetic states are the ground states of every two-spin Hamiltonian

Consider

$$
H_{11}=-4 J\left(S_{1}^{x} S_{2}^{x}+S_{1}^{Y} S_{2}^{y}+\gamma S_{1}^{z} S_{2}^{z}\right) .
$$

The eigenvectors and their corresponding eigenvalues $\varepsilon$ are given by:

$$
\begin{aligned}
& 2^{-1 / 2}[|+-\rangle-|-+\rangle] \quad \varepsilon=(\gamma+2) J, \\
& 2^{-1 / 2}[|+-\rangle+|-+\rangle] \quad \varepsilon=(\gamma-2) J, \\
& \left.\begin{array}{l}
1++\rangle \\
1--\rangle
\end{array}\right\} \\
& \varepsilon=-\gamma J,
\end{aligned}
$$

in which $\mid+->$ denotes a state with spin up on the first site, and spin down on the second one, etc. It is immediately clear that the two ferromagnetic states have the lowest energy.

Summing over the contributions of all two-spin Hamiltonians, we obtain a lower bound for $E_{0}$ :

$$
E_{0} \geqslant 2 \sum_{\delta} \sum_{\rho(\delta)}(-\gamma J)=2.3 \frac{N}{2}(-\gamma J)=-3 N \gamma J
$$


The factor 2 arises from the fact that $\Sigma_{\delta} H_{1 \delta}$ and $\Sigma_{\delta} H_{2 \delta}$ give the same contribution to the lower bound of $E_{0}$. On the other hand,

$$
E_{0} \leqslant-3 N \gamma J
$$

is obtained by noting that $\left|F_{+}\right\rangle,\left|F_{-}\right\rangle$are eigenstates of $H$ with energy $-3 N \gamma J$. Hence,

$$
E_{0}=-3 N \gamma J \text {. }
$$

The theorem can now be applied in two steps:

4.1.1. First step. The energy gap $\Delta(\boldsymbol{\delta})$ of each $H_{\delta}$ is estimated with the help of the subdivision:

$$
H_{\delta}=H_{1 \delta}+H_{2 \delta}
$$

The specific character of the ferromagnetic ground states excludes the existence of any other common ground state. The energy gap $\Delta(\delta)$ can then be estimated very easily. Indeed, remembering that $H_{1 \delta}$ and $H_{2 \delta}$ describe two systems of non-interacting spin pairs, the non-common ground states of which are obviously mutually orthogonal, we may put $x=0$ in (10) and conclude that the gap in the spectrum of $H_{1 \delta}$ is a lower bound for $\Delta(\boldsymbol{\delta})$. We obtain one of the lowest-lying (degenerate!) excited states of $H_{1 \delta}$ by taking $(N / 2-1)$ pairs in their ground state $(|++\rangle$ or $|--\rangle)$ and only one pair in the state

$$
2^{-1 / 2}[|+-\rangle+|-+\rangle]
$$

If the ground-state energy of $H_{1 \delta}$ is subtracted from the energy of a lowest-lying excited state, we obtain the desired lower bound:

$$
\Delta(\boldsymbol{\delta}) \geqslant(\gamma-2) J+\left(\frac{1}{2} N-1\right)(-\gamma J)-\frac{1}{2} N(-\gamma J)=2(\gamma-1) J
$$

4.1.2. Second step. The total gap of $H$ is estimated with the help of the subdivision:

$$
H=\sum_{\delta} H_{\delta}
$$

Also now all non-common ground states of the different $H_{\delta}$ are mutually orthogonal. Putting now $x=0, \Delta=\Delta(\boldsymbol{\delta})$ in (11), we obtain finally:

$$
E_{1}-E_{0} \geqslant 3(\gamma-1) J
$$

The gap has a vanishing lower bound if $\gamma \rightarrow 1$, according to the exact behaviour of the low-lying energy spectrum of an isotropic Heisenberg ferromagnet.

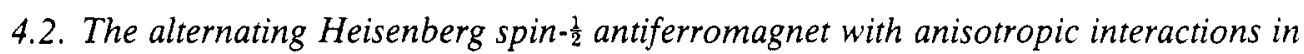
one dimension

The Hamiltonian for this system is taken to be

$H=\sum_{\alpha=x, y, z} H_{\alpha}$

$H_{\alpha}=\sum_{j=1}^{N}\left[4 J_{\alpha}\left(S_{2 j-1}^{\alpha} S_{2 j}^{\alpha}+\gamma S_{2 j}^{\alpha} S_{2 j+1}^{\alpha}\right)+4 \gamma\left(J_{1 \alpha} S_{2 j-1}^{\alpha} S_{2 j+1}^{\alpha}+J_{2 \alpha} S_{2 j}^{\alpha} S_{2 j+2}^{\alpha}\right)\right]$,

$J_{\alpha}, J_{1 \alpha}, J_{2 \alpha}>0 \quad 0 \leqslant \gamma<1$

$J_{1 \alpha}+J_{2 \alpha}=J_{\alpha} \quad J_{\alpha}>2 \gamma J_{1 \alpha} \quad J_{\alpha}>2 \gamma J_{2 \alpha} \quad \alpha=1,2$. 
The ground state of $H$ is found to be the singlet-pair state (sPS), given by (Caspers 1982 , Caspers and Magnus 1982, 1983, Van den Broek 1980)

$$
\left|\varphi_{0}\right\rangle=2^{-N / 2} \prod_{j=1}^{N}[|+-\rangle-|-+\rangle]_{j} .
$$

Following Caspers and Magnus $(1982,1983)$ and Van den Broek (1980) it is easy to show that the conditions formulated in (20) are sufficient to prove that $\left.\mid \varphi_{0}\right)$ is an eigenstate of $H$ and of each $H_{\alpha}$ :

$$
\begin{aligned}
& H\left|\varphi_{0}\right\rangle=-N\left(J_{x}+J_{y}+J_{z}\right)\left|\varphi_{0}\right\rangle \\
& H_{\alpha}\left|\varphi_{0}\right\rangle=-N J_{\alpha}\left|\varphi_{0}\right\rangle \quad \alpha=x, y, z .
\end{aligned}
$$

Let $T$ represent a translation over two lattice spacings:

$$
\begin{array}{lr}
T S_{l} T^{+}=S_{i+2} & 1 \leqslant i \leqslant 2 N-2 \\
T S_{2 N-1} T^{+}=S_{1} & T S_{2 N} T^{-}=S_{2} .
\end{array}
$$

Then it is clear that $[H, T]=0$. Therefore, a convenient subdivision of $H_{\alpha}$, according to

$$
H_{\alpha}=H_{1 \alpha}+H_{2 \alpha},
$$

where $H_{2 \alpha}=T H_{1 \alpha} T^{+}$, and $H_{1 \alpha}$ describes a set of $\frac{1}{2} N$ non-interacting cells of four spins, is obtained by putting

$$
\begin{aligned}
& H_{1}=\sum_{j=1}^{N / 2} H_{1 j \alpha} \\
& H_{11 \alpha}=2 J_{\alpha}\left(S_{1}^{\alpha} S_{2}^{\alpha}+2 \gamma S_{2}^{\alpha} S_{3}^{\alpha}+S_{3}^{\alpha} S_{4}^{\alpha}\right)+4 \gamma\left(J_{1 \alpha} S_{1}^{\alpha} S_{3}^{\alpha}+J_{2 \alpha} S_{2}^{\alpha} S_{4}^{\alpha}\right), \text { etc. }
\end{aligned}
$$

The coupling strengths between spins on sites 1,2 and 3,4 (and also on the corresponding sites in other cells) are $2 J_{\alpha}$ instead of $4 J_{\alpha}$ because all those bonds also appear in $H_{2 \alpha}$. As a consequence, they are counted twice. We first prove the existence of a common ground state of $H$ and all $H_{\alpha}$. which, in this case, turns out to be the SPS. This implies:

$$
\begin{aligned}
& E_{0 \alpha}=-N J_{\alpha} \quad \alpha=x, y, z \\
& E_{0}=-N\left(J_{x}+J_{y}+J_{z}\right) .
\end{aligned}
$$

To prove (22), we first remark that we have from (21), for $\alpha=x, y, z$ :

$$
E_{0 \alpha} \leqslant-N J_{\alpha} \quad E_{0} \leqslant-N\left(J_{x}+J_{y}+J_{z}\right) .
$$

To be specific, we take $\alpha=z$. Every Ising state (i.e. a state containing spins up and/or down with respect to $\boldsymbol{e}_{z}$ ) is an eigenstate of $H_{11 z}$. Careful inspection allows us to write down the ground states and lowest-lying excited states of $H_{11 z}$, with their corresponding energies $\varepsilon$ :

$$
\left.\begin{array}{l}
|++-+\rangle \\
|--+-\rangle
\end{array}\right\} \varepsilon=-2 \gamma J_{1 z}
$$




$$
\left.\begin{array}{l}
\left.\begin{array}{l}
|+-++\rangle \\
1-+--\rangle
\end{array}\right\} \varepsilon=-2 \gamma J_{2 z} \\
\begin{array}{l}
++-+\rangle \\
1-++-\rangle \\
1+-+-\rangle \\
|-+-+\rangle
\end{array}
\end{array}\right\} \varepsilon=-J_{z} \text { (ground state) }
$$

Taking into account the contributions from both $H_{1 z}$ and $H_{2 z}$, we find:

$$
E_{0 z} \geqslant 2 \times(N / 2)\left(-J_{z}\right)=-N J_{z} .
$$

Of course, an analogous result follows for $\alpha=x, y$. Hence,

$$
\begin{aligned}
& E_{0 \alpha} \geqslant-N J_{\alpha} \quad \alpha=x, y, z \\
& E_{0} \geqslant-N\left(J_{x}+J_{y}+J_{z}\right)
\end{aligned}
$$

and (22) follows from (23) and (25).

Next, the existence of an energy gap is shown by threefold application of our theorem:

4.2.1. There is a gap in the spectrum of $H_{\alpha}$, denoted by $\Delta_{\alpha}, \alpha=x, y, z$. For convenience, we put $K_{\alpha}=\max \left(J_{1 \alpha}, J_{2 \alpha}\right), \alpha=x, y, z$. Take $\alpha=z$. The lowest-lying excited states of $H_{11 z}$ have an energy $-2 \gamma K_{z}$. Furthermore, because we are only dealing with Ising states, any two non-common ground states of $H_{1 z}$ and $H_{2 z}$ are mutually orthogonal, because they have at least one site with different spin orientations. So we may put $x=0, \Delta=$ $J_{z}-2 \gamma K_{z}$ in (10) to find:

$$
\Delta_{z} \geqslant J_{z}-2 \gamma K_{z} \text {. }
$$

Of course, the same conclusion holds for the other two gaps $\Delta_{x}, \Delta_{y}$. So:

$$
\Delta_{\alpha} \geqslant J_{\alpha}-2 \gamma K_{\alpha} \quad \alpha=x, y, z .
$$

4.2.2. There is a gap $\Delta_{x z}$ in the spectrum of $H_{x}, H_{z}$, where

$$
\Delta_{x z} \geqslant \min \left(\Delta_{x}, \Delta_{z}\right)
$$

To prove this, we have to show that the scalar product of any two non-common ground states of $H_{z}$ and $H_{x}$ vanishes. It can be seen easily that

$$
R S_{i}^{z} S_{j}^{z} R^{+}=S_{i}^{x} S_{j}^{x} \quad 1 \leqslant i, j \leqslant 2 N
$$

where

$$
R=\prod_{r=1}^{2 N}\left[2^{-1 / 2}\left(1-\mathrm{i} \sigma_{r}^{y}\right)\right]
$$

rotates every spin vector in $\mathscr{H}$ over an angle $+\pi / 2$ around $e_{y}$, and $\boldsymbol{\sigma}_{r}^{v}$ is a Pauli matrix, defined on site $r$. Therefore, all ground states of $H_{x}$ are generated by applying $R$ on the ground states of $H_{z}$.

Denote by $M$ the matrix built up by all possible scalar products of ground states of $H_{z}$ with ground states of $H_{x}$. Then $M$ is the matrix representing the projection of $R$ onto the subspace $\mathscr{H}_{0 z}$. With the help of the four-spin-cell spectrum, we compare the different 
eigenvalues of $H_{z}$ with $E_{0 z}$. It turns out that $E_{0 z}$ is reached only by Ising states containing on each site pair $(2 j-1,2 j) \mathrm{a}|+-\rangle-$ or $|-+\rangle-$ configuration. This allows us to write $M$ as an outer product of $N$ two-spin matrices:

$$
A=\mu \times \mu \times \ldots \mu \text { ( } N \text { factors }) .
$$

$\mu$ is, of course, the projection of $2^{-1 / 2}\left(1-i \sigma_{1}^{\gamma}\right) 2^{-1 / 2}\left(1-i \sigma_{2}^{y}\right)$ onto the truncated basis $\{|+-\rangle,|-+\rangle\}$. The result is

$$
\mu=\left[\begin{array}{rr}
1 / 2 & -1 / 2 \\
-1 / 2 & 1 / 2
\end{array}\right] \text {. }
$$

The eigenvalues of $\mu$ are 0 and 1 . Obviously, the eigenvalues of $M$ are 1 and 0 with multiplicities 1 and $2^{N}-1$ respectively. The eigenvalue 1 corresponds to the singlet configuration $2^{-1 / 2}[|+-\rangle-|-+\rangle]$ for each spin pair, i.e. to the sPS, the only common ground state of $H_{x}$ and $H_{z}$.

Now, any scalar product of non-common ground states is bounded by the largest eigenvalue of $M$, which does not correspond to the SPS. This particular eigenvalue is found to be zero. Therefore we may use the theorem in the sense of $\S 3.2$, if we put $x=0, \Delta=\min \left(\Delta_{x}, \Delta_{z}\right)$ in (18). This completes the proof of (27).

\subsubsection{There is a gap in the energy spectrum of $H$. Using the subdivision}

$$
H=\left(H_{x}+H_{z}\right)+H_{y}
$$

and remembering from (28) that $\left|\varphi_{0}\right\rangle$ is the non-degenerate ground state of $H_{x}+H_{z}$, we may apply the theorem again according to $\$ 3.2$. Indeed, since $H_{x}+H_{z}$ and $H_{y}$ have no other than common ground states, we may put $x=0, \Delta=\min \left(\Delta_{x z}, \Delta_{y}\right)$ in (18) to obtain finally:

$$
\begin{aligned}
& E_{1}-E_{0} \geqslant \min \left(\Delta_{x z}, \Delta_{y}\right) \geqslant \min _{\alpha=x, y, z} \Delta_{\alpha} \\
& \Delta_{\alpha}=J_{\alpha}-2 \gamma K_{\alpha}>0 \quad \alpha=x, y, z .
\end{aligned}
$$

It should be remarked, however, that a better lower bound for $E_{1}-E_{0}$ can be obtained if all interactions are isotropic, i.e.

$$
J_{\alpha}=J \quad J_{1 \alpha}=J_{1} \quad J_{2 \alpha}=J_{2} \quad \alpha=x, y, z \quad K=\max \left(J_{1}, J_{2}\right) .
$$

In that case we have $T H_{1} T^{+}=H_{2}$, etc, where $T$ generates a cyclic permutation of $H_{1}$, $\mathrm{H}_{2}, \mathrm{H}_{3}$. As a consequence we may conclude immediate from (11):

$$
E_{1}-E_{0}>\frac{3}{2}(J-2 \gamma K) \text {. }
$$

It should be noted that, in general, the lower bound for the energy gap, as it is estimated by the theorem, is smaller than the exact energy gap. For example, take $\gamma=0$ in (29). Then $E_{1}-E_{0} \geqslant \frac{3}{2} J$. Now $H$ describes a system of non-interacting spin pairs. The gap in the energy spectrum equals $4 J$ in this case.

\section{Discussion}

Unfortunately, the class of quantum systems whose exact ground state is known explicitly is rather small, although it has been extended recently (Caspers 1982, Caspers and 
Magnus 1983, Klein 1982, Van den Broek 1980) One of the quantum spin systems treated in Caspers (1982) and Caspers and Magnus (1983) is obtained if we take in our example the limits $\gamma \rightarrow 1, J_{1 \alpha}, J_{2 \alpha} \rightarrow \frac{1}{2} J_{\alpha}$ in $\S 4.2$. Apparently, the lower bound of the energy gap tends to zero. This, however, does not lead us to any conclusion about the existence of an energy gap in the spectra of those systems.

In particular, a subdivision according to $\$ 4.2$ would introduce an analogous $M$ matrix. It turns out that the double degeneracy of the ground state gives rise to serious computational problems if the eigenvalues are to be calculated rigorously.

During the last fifteen years, many attempts have been made (Caspers and Magnus 1982, Majumdar 1970, Majumdar et al 1972, Shastry and Sutherland 1981) to obtain information about the energy spectrum of Hamiltonians of the type:

$$
H=4 \sum_{i=1}^{2 N} S_{i} \cdot S_{i+1}+2 \sum_{i=1}^{2 N} S_{i} \cdot S_{i-2} .
$$

No way has yet been found to demonstrate exactly whether there exists an energy gap or not.

On the other hand, the system described by the Hamiltonian of $\S 4.2$ is a non-trivial example of an alternating dimerised linear antiferromagnet, for which the existence of an energy gap has been proved in this paper. Although it has been conjectured by many authors that dimerisation always leads to a gap in the energy spectrum, up to now no one has been able to prove rigorously the existence of such a gap in the usual nearestneighbour alternating Heisenberg linear chain $\left(J_{1 \alpha}=J_{2 \alpha}=0\right.$ in $\left.\$ 4.2\right)$, which remains an interesting quantum system either as a basic model for a class of spin-Peierls transitions, or in connection with the study of organic free radicals (Bonner and Blöte 1982, Bonner et al 1983).

\section{References}

Bonner J C and Blöte H W J 1982 Phys. Rev. B 256959

Bonner J C, Friedberg S A, Kobayashi H, Meier D L and Blöte H W J 1983 Phys. Rev. B 27248

Caspers W J 1982 Physica 115A 275

Caspers W J and Magnus W 1982 Phys. Lett. 88A 103

- 1983 Physica 119A 291

Klein D J 1982 J. Phys. A: Math. Gen. 15661

Majumdar C K 1970 J. Phys. C. Solid State Phys. 3911

Majumdar C K, Krishnan K and Mubayi V 1972 J. Phys. C: Solid State Phys. 52896

Shastry B S and Sutherland B 1981 Phys. Rev. Lett. 47964

Van den Broek P M 1980 Phys. Lett. 77A 261 